\title{
A comparison of droplet and contact contamination using 3 simulated barrier techniques for COVID-19 intubation: a quality assurance study
}

\author{
Peter Rose MD MSc, John Veall MD, Navraj Chima MD, Elena Vowels BMBS, Shruti Chitnis MBChB, \\ Alana Flexman MD, Raymond Tang MD MSc
}

\section{Abstract}

Background: The intubation of patients with coronavirus disease 2019 (COVID-19) puts health care workers at risk of infection through aerosol, droplet and contact contamination. We evaluated the risk of droplet and contact contamination for health care workers using 3 intubation barrier techniques as part of a quality assurance study at our institution.

Methods: This randomized quality assurance study was completed at a tertiary academic hospital in Vancouver, British Columbia, Canada, on Apr. 4, 2020. Participants in personal protective equipment performed simulated intubations on a manikin with (a) no barrier, (b) a clear plastic sheet covering the manikin and (c) a plexiglass intubation box over the manikin, in random order. Fluorescein was ejected from inside the manikin's mouth to simulate droplet and contact spread during a standard intubation sequence. Two blinded independent assessors evaluated the location and degree of contamination on the intubator and assistant using an ultraviolet light. Contamination severity was rated in a standard fashion ( $0=$ none; $1=$ minor; $2=$ major). The primary outcome was total contamination score and secondary outcomes were scores between intubator and assistant, anatomic areas contaminated and qualitative feedback on ease of intubation.

Results: Five participants completed this study. Total contamination score was different between the 3 groups for the intubator $(p=0.02)$ but not the assistant $(p=0.2)$. For the intubator, the total contamination score was higher when the sheet was used (median 29 [interquartile range (IQR) 25-34]) than when the box was used (median 17 [IQR 15-22]) or when no barrier was used (median 18 [IQR 13-21]). All 5 participants reported challenges during intubation using the sheet.

Interpretation: Use of a plastic sheet while intubating patients with COVID-19 may increase the risk of droplet and contact contamination during intubation and impede intubation. Further study should be undertaken before implementing barrier techniques in practice.

oronavirus disease 2019 (COVID-19) is spread by droplet and contact contamination and through viral aerosolization during aerosol-generating medical procedures such as intubation and bag-valve-mask (BVM) ventilation. ${ }^{1-3}$ Previous research has demonstrated that health care workers are at higher risk of viral transmission during these aerosol-generating medical procedures. ${ }^{1-3}$ During the COVID-19 pandemic, the heightened need to protect health care workers as well as shortages in personal protective equipment (PPE) have led to a rapid proliferation of improvised, repurposed and innovative techniques to minimize exposure. . $^{4-9}$

Given that $46 \%-90 \%$ of health care workers have been shown to self-contaminate while doffing (removing) PPE, contamination of gowns and equipment may be an important source of infection of health care workers after they perform airway management procedures. ${ }^{10-12}$ Potential solutions under consideration at our institution included the use of a clear plastic drape and the use of a plexiglass box around the patient's head as a barrier during intubation. Both of these techniques have been described on social media and have been adopted in many centres, despite limited evidence that they reliably reduce contamination. ${ }^{6-9}$

The protocol at our institution for intubation of patients with suspected COVID-19 has prioritized rapid airway establishment with only essential personnel, to limit the exposure of health care workers to the virus. Consistent with other guidelines, preoxygenation in a negative-pressure room is followed by rapid administration of induction

Competing interests: None declared.

Correspondence to: Peter Rose, peter.rose3@vch.ca

CMAJ Open 2020. DOI:10.9778/cmajo.20200090 
agents, minimal BVM ventilation and intubation with video laryngoscopy. ${ }^{13,14}$ Our current practice is to place a plastic sheet over the patient's head immediately following induction to reduce contamination, but our clinical impression is that this technique results in additional contamination and is an impediment to intubation. In addition, it remains unclear whether this is the best approach.

After development of a plexiglass intubation box at the University of British Columbia, we aimed to conduct a systematic comparison of these techniques to inform our institutional protocols for the intubation of patients with COVID-19. Our primary objective was to compare contact contamination of personnel using 3 techniques (a plastic sheet, a plexiglass box and no barrier) with a simulated model of droplet contamination during intubation. Our secondary objective was to compare contamination between the intubator and the assistant and to do a qualitative assessment of both the anatomic range of contamination and the ease of intubation using each technique.

\section{Methods}

\section{Setting and design}

This study was conducted on Apr. 4, 2020, at Vancouver General Hospital, a quaternary academic hospital serving a population of 5 million people, in Vancouver, British Columbia, Canada. It was a randomized quality assurance study.

\section{Study population}

Participants were 5 anesthesiologists selected among staff and fellows in the Department of Anesthesiology and Perioperative Care who were involved in preparing the institutional protocol for the intubation of patients with COVID-19. Both male and female participants with a range of heights were selected to participate. All participants were experienced in endotracheal intubation and were familiarized with the manikin (i.e., model employed in medical simulation) before starting the study.

Each anesthesiologist performed the study procedure using each of the 3 techniques (described later), resulting in 15 trials. For each trial, another participant who was available at that time acted as the assistant; assistants were not consistently paired with the same intubators. The order of trials for each intubator was assigned randomly immediately before the start of the trial.

\section{Procedures}

A simulated intubation protocol was developed by consensus (P.R., J.V., A.F., R.T.), consistent with national and international COVID-19 airway management recommendations, ${ }^{13,14}$ to compare a no-barrier technique with 2 barrier techniques for use during COVID-19 intubations at our institution. Participants performed 3 simulated intubations using 3 techniques in random order: control (no additional protective devices); use of a plastic sheet over the patient; and use of a plexiglass intubation box. Before each procedure, the intubator and assistant donned the standardized
PPE, which consisted of a gown, nitrile nonsterile gloves, a surgical mask, a full face shield and a disposable head covering. N95 respirators were not used in this study to preserve the supply for clinical use. Before each procedure, both participants were inspected with an ultraviolet (UV) light to ensure that there was no fluorescing material on the PPE at baseline.

An intubation manikin head was equipped with a MADgic laryngo-tracheal mucosal atomization device (Teleflex) directed out of the mouth with the nozzle situated at the teeth as described previously. ${ }^{15}$ The technique was modified by J.V. (the simulation lead for our residency program) using fluorescein (Akorn Pharmaceuticals) instead of fluorescent powder to ensure that the level of droplets produced in each trial was reliable and reproducible. The MADgic device produces droplets in the 70 - to $100-\mu \mathrm{m}$ range and was tested before the study to ensure reproducible dispersion. This device was connected to an infusion line and infusion pump (B. Braun Medical) under the bed.

With the intubator standing at the head of the bed and the assistant to the right of the manikin, $5 \mathrm{~mL}$ of fluorescein $2 \mathrm{mg} / \mathrm{mL}$ was injected through the MADgic device in a standardized fashion over 22 seconds to simulate surface contamination present before intubation. A BVM (Ambu) was then placed over the mouth and nose by the intubator and the fluorescein injection continued to simulate contamination during preoxygenation. The BVM was then held in place for a further 60 seconds to simulate the time required to wait for the onset of paralysis during the recommended rapid sequence induction of anesthesia. Following this, the intubator proceeded to intubate with a styletted $6.5-\mathrm{mm}$ size endotracheal tube using a McGrath video laryngoscope (Medtronic). Once the endotracheal cuff was inflated and the endotracheal tube was connected to the BVM, the trial was considered complete, and the 2 participants (intubator and assistant) stepped away from the manikin for evaluation by the assessors, as described below.

After each trial procedure, the manikin and all equipment were thoroughly cleaned and visually inspected with a UV light to ensure adequate removal of fluorescein. The PPE donned by the intubator and assistant were changed between trials. At the end of the study, qualitative comments about the ease of intubation with each technique were solicited from the participants.

\section{Plastic sheet}

In the plastic sheet study arm, a large, clear plastic sheet (120 cm wide $\times 150 \mathrm{~cm}$ long) was placed on the bed in line with the shoulder of the manikin. After the simulated preoxygenation, the sheet was brought over the head of the manikin and intubation was performed with the arms of the intubator and assistant under the sheet.

\section{Plexiglass box}

The intubating plexiglass box was created from clear acrylic that was one-eighth of an inch thick (Figure 1, CovidBox version 2.3, CovidBox, Vancouver, BC). In the plexiglass box study 


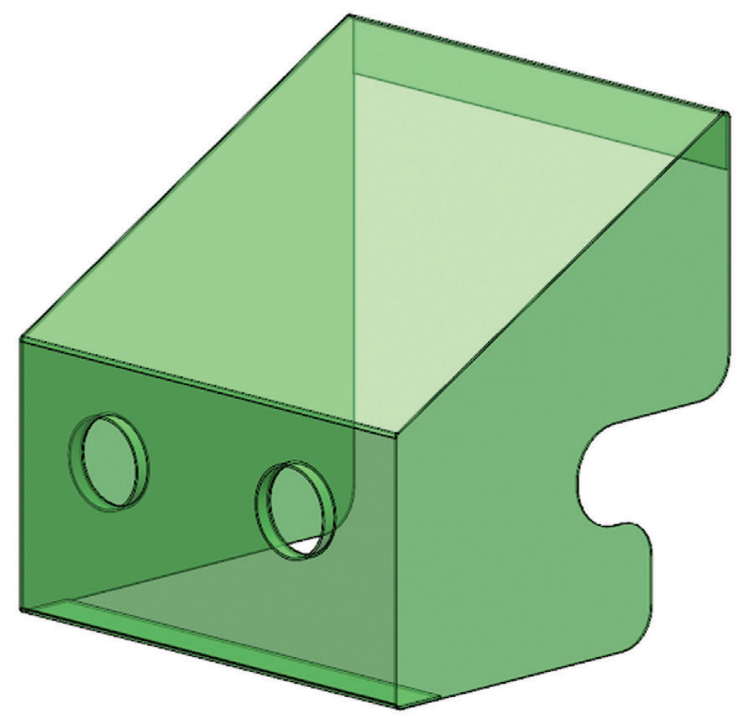

Figure 1: Plexiglass intubation box (CovidBox version 2.3 [CovidBox, Vancouver, $\mathrm{BC}]$ ). The intubator performs the intubation through the 2 holes while the assistant helps using their arm along the notch on the right. The patient's head is positioned within the intubation box and directed toward the intubator's arm holes. The patient's torso is directed away from the intubator.

arm, the plexiglass box was placed over the manikin head before the study procedures were started, and the intubator performed the simulated preoxygenation and intubation by accessing the manikin head through 2 holes in the box. The assistant accessed the manikin head through cutouts on the right side of the box.

\section{Outcome measures}

After each trial, 2 independent assessors, blinded to the technique used and to the role each participant had performed (intubator or assistant), scored the degree of contamination on both the intubator and the assistant using a UV light in a dark room on a standardized scale $(0=$ no contamination, $1=$ light contamination, 2 = heavy contamination) (Figure 2). The contamination assessment was based on previously described methods for grading contamination in the operating room, except that parts of the body rather than items in the operating room were assessed. ${ }^{16}$ The following parts of the body were assessed, each of which was scored separately: hand, forearm, upper arm, head, neck, torso, legs and feet. Both the front and back of each body part were assessed.

The primary outcome was the total contamination score summed from all anatomic areas and both independent assessments. Secondary outcomes were the scores of the intubators and the assistants, the anatomic areas that were contaminated and the participants' qualitative comments about the ease of intubation with each technique.

\section{Statistical analysis}

A sample size of convenience of 5 participants was chosen because of time and resource constraints during the

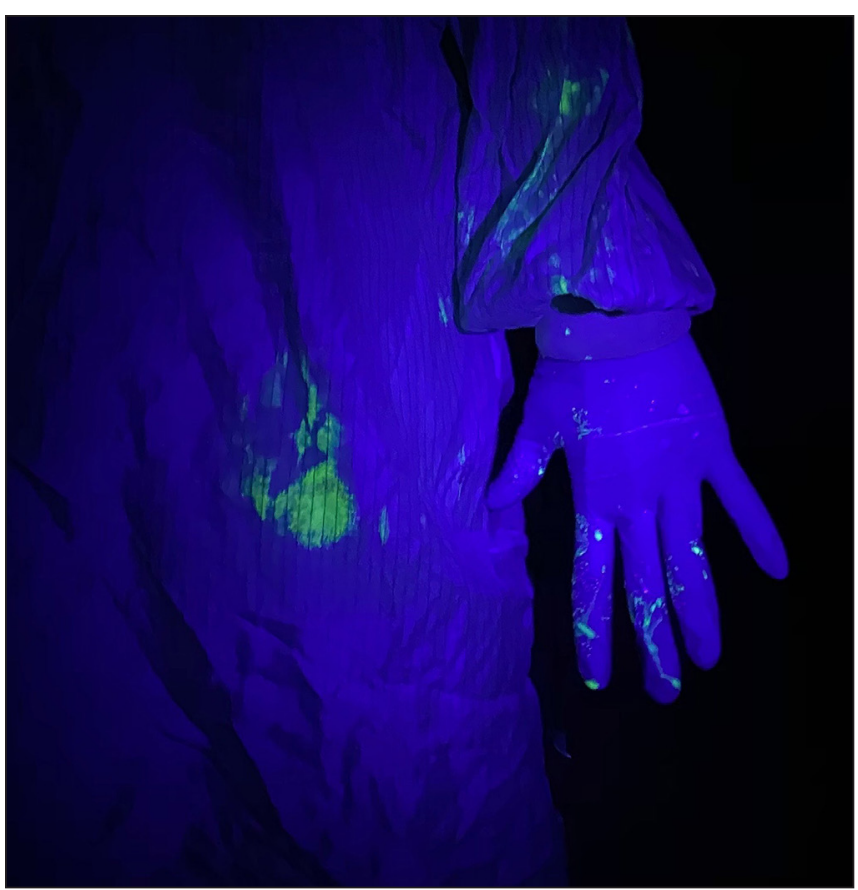

Figure 2: Intubator heavily contaminated with fluorescein on hand, forearm and torso highlighted with ultraviolet light after performing an intubation on a manikin covered by a plastic sheet.

pandemic. Data were described using percentages, means with standard deviations (SDs), medians with interquartile ranges (IQRs) and ranges, as appropriate. Differences in total score across the 3 study arms were analyzed using a Friedman test for repeated measures on each subject. If significant $(p<0.05)$, a post-hoc Wilcoxon signed-rank test for paired data was used to compare pairs with a Bonferroni correction to account for multiple comparisons, where a $p$ value less than 0.02 was considered statistically significant. A Wilcoxon rank-sum test was used to compare total scores between intubators and assistants and a Spearman correlation coefficient was used to determine the correlation between the 2 assessors. All data analysis was performed using Stata version 12.1 (StataCorp) and a $p$ value less than 0.05 was considered statistically significant unless otherwise specified.

\section{Ethics approval}

After review by the UBC Clinical Research Ethics Board, requirements for formal approval and informed consent were waived as the study was considered a quality assurance project.

\section{Results}

Three women and 2 men participated in the study. The mean height was $174 \mathrm{~cm}$ (range 159-190 cm) (Table 1).

Scores for contamination of each body part, stratified by role and presented as the sum of the scores for the 2 assessors, are provided in Table 2. Most contamination was limited to the hands, arms and chests of the participants. One intubator had light contamination of the head and neck, and another experienced light contamination of the lower body; 


\begin{tabular}{|lcccc|}
\hline \multicolumn{5}{|l|}{ Table 1: Characteristics of the study participants } \\
\hline Participant & Age, yr & Sex & Height, cm & Weight, kg \\
\hline 1 & 38 & Male & 190 & 80 \\
\hline 2 & 36 & Female & 183 & 91 \\
\hline 3 & 47 & Male & 173 & 63 \\
\hline 4 & 33 & Female & 159 & 50 \\
\hline 5 & 41 & Female & 165 & 62 \\
\hline Mean \pm SD & $39 \pm 5$ & & $174 \pm 12$ & $69 \pm 16$ \\
\hline SD = standard deviation. & & & & \\
\hline
\end{tabular}

Table 2: Contamination scores

\begin{tabular}{|c|c|c|c|c|c|c|}
\hline \multirow[b]{2}{*}{ Location } & \multicolumn{3}{|c|}{ Intubator; study arm } & \multicolumn{3}{|c|}{ Assistant; study arm } \\
\hline & Control & Plastic sheet & Plexiglass box & Control & Plastic sheet & Plexiglass box \\
\hline Right hand & $5(5-5)$ & $7(7-7)$ & $7(7-7)$ & $2(2-2)$ & $5(4-6)$ & $3(3-6)$ \\
\hline Left hand & $6(6-7)$ & 7 (7-8) & $8(7-8)$ & $3(2-4)$ & $6(5-7)$ & $4(3-5)$ \\
\hline Right forearm & $0(0-0)$ & $5(5-5)$ & $1(0-3)$ & $0(0-0)$ & $0(0-3)$ & $0(0-0)$ \\
\hline Left forearm & $3(2-4)$ & $5(4-6)$ & $2(0-3)$ & $0(0-0)$ & $0(0-2)$ & $0(0-0)$ \\
\hline Right upper arm & $0(0-0)$ & $0(0-2)$ & $0(0-0)$ & $0(0-0)$ & $0(0-0)$ & $0(0-0)$ \\
\hline Left upper arm & $0(0-0)$ & $0(0-0)$ & $0(0-0)$ & $0(0-0)$ & $0(0-0)$ & $0(0-0)$ \\
\hline Torso & $0(0-2)$ & $3(2-4)$ & $0(0-0)$ & $0(0-0)$ & $0(0-0)$ & $0(0-0)$ \\
\hline Head and neck & $0(0-0)$ & $0(0-0)$ & $0(0-0)$ & $0(0-0)$ & $0(0-0)$ & $0(0-0)$ \\
\hline Lower body & $0(0-0)$ & $0(0-0)$ & $0(0-0)$ & $0(0-0)$ & $0(0-0)$ & $0(0-0)$ \\
\hline Total score & $18(13-21)$ & $29(25-34)$ & $17(15-22)$ & $4(4-6)$ & $11(10-16)$ & $8(7-9)$ \\
\hline
\end{tabular}

both of these events occurred in the control study arm. The total contamination scores were highly correlated between the 2 assessors (coefficient $\rho=0.7841, p<0.001$ ). Complete contamination data for each intubation trial are provided in Appendix 1 (available at www.cmajopen.ca/content/8/3/ E554/suppl/DC1).

The total contamination score was statistically different between the 3 study arms for the intubator $\left(\chi^{2}=7.5, p=0.02\right)$ but not the assistant $\left(\chi^{2}=3.6, p=0.2\right)$. For the intubator, the total contamination score was higher when the plastic sheet was used than when no barrier was used (median 29 [IQR 25-34] v. 18 [IQR 13-21], $p=0.04$ ), and it was higher when the plastic sheet was used than when the plexiglass box was used (median 29 [IQR 25-34] v. 17 [IQR 15-22], $p=0.04$ ), but there was no difference between the control study arm versus the plexiglass box study arm (18 [IQR 13-21] v. 17 (IQR 15-22), $p=0.9$ ). The differences in the first 2 comparisons were found not to be statistically significant after correction for multiple comparisons $(p<0.02)$.

Participants performing intubation had higher total contamination scores than those acting as assistants (median 21 [IQR 15-25] v. 9 [IQR 6-11], respectively, mean difference 12 [95\% confidence interval 6-17], $p<0.001)$.
All 5 participants recorded narrative comments about the intubating conditions with the plastic sheet or plexiglass box compared with no barrier (narrative comments are transcribed in Appendix 2, available at www.cmajopen.ca/ content/8/3/E554/suppl/DC1). Four participants reported that it was challenging to visualize the video laryngoscope screen using the sheet and all participants reported challenges or difficulty intubating using the sheet compared with no barrier. One participant reported altered visualization of the video laryngoscope screen using the box and 3 participants reported some spatial or positioning limitations during intubation with the box compared with no barrier.

\section{Interpretation}

Our study results demonstrate that contact and droplet contamination during a simulated airway intervention differed among the 3 barrier techniques we tested (no barrier, clear plastic sheet, plexiglass box). The intubator experienced higher rates of contamination than the assistant, and contamination of the head and lower body was only observed with the no-barrier technique. All participants subjectively reported difficulty performing intubation 
using the plastic sheet. Overall, the plastic sheet does not appear to provide any benefit over the other techniques and may increase contact contamination while impairing intubation, although our results must be interpreted cautiously given the small sample size and the limited generalizability of the results.

As a result of the COVID-19 pandemic, many airway management protocols have incorporated barriers to reduce viral spread through aerosolization and droplets, although few of these techniques have been studied and none have been formally incorporated in recommendations. Use of a plastic sheet covering the patient's head was introduced at our institution as a means to reduce droplet and aerosol transmission during COVID-19 intubations, ${ }^{6,9}$ and an intubating box was explored as an alternative barrier. ${ }^{7,8}$

Although the benefits of barriers such as a sheet have been demonstrated in awake patients and during extubation, ${ }^{6}$ the benefits are less clear during intubation. In addition, the sheet is not typically placed over patients with respiratory compromise while they are awake, which may lead to further droplet contamination when the sheet is advanced during intubation. The intubation box used in our study was subjectively easier to use but presents challenges in transportation and decontamination, which were not examined in our study.

Our results contribute to the literature on droplet and contact contamination during simulated intubation. As a result of our study and recently published work showing increased exposure to airborne particles and increased time for intubation with use of an intubation box, ${ }^{17,18}$ our institution has changed to a no-barrier technique.

The risk of contact contamination during intubation is substantial as the aerosol and droplet particles settle on surfaces and may be concentrated around the head of the patient. In addition, our simulated model may underestimate the degree of contamination if the patient has been in the environment for a long time. The plastic sheet may act as a medium to transfer contaminants to health care workers and although it may reduce aerosol and droplet spread by coughing patients, ${ }^{6}$ the benefit during intubation in an apneic patient not receiving BVM ventilation is unclear.

\section{Limitations}

Our study has several limitations and our results should be interpreted cautiously. We enrolled a small number of participants and conducted a limited number of trials. In addition, our results may not be generalizable to people of different sizes or levels of training or who use different intubation techniques. We used an unvalidated simulated intubation model using a manikin that may not reflect clinical practice or actual droplet contamination, although it was similar to previously published models and we attempted to mirror clinical practice in every other way. The assessment scale we used was practical but not validated, and the significance of the total score is not established. We chose the total contamination score as our primary outcome, and the significance of this score compared with individual anatomic areas is unknown. Finally, given the nature of the study, the intubators could not be blinded to group allocation (i.e., control, sheet or box), which could have been an inadvertent source of bias.

\section{Conclusion}

Overall, our study results suggest that barrier techniques designed to reduce droplet and contact contamination, specifically a plastic drape used as described in our study, may actually increase contamination. Intubation barrier devices require further study and validation before they are introduced into widespread practice. Not only are the benefits unclear, subjective feedback in our study suggests that these barriers may impede intubation. A larger trial would be useful to evaluate the use of novel devices for their risk modification of aerosol, droplet and contact contamination as well as the need for additional steps that may delay airway securement and prolong the exposure of health care workers. Large multicentre registries such as IntubateCOVID (www. intubatecovid.org) that collect data on the use of different types of barriers may help to inform practice in the future.

\section{References}

1. Schwartz J, King C-C, Yen M-Y. Protecting health care workers during the COVID-19 coronavirus outbreak - lessons from Taiwan's SARS response. Clin Infect Dis 2020;71:858-60.

2. Wu Z, McGoogan JM. Characteristics of and important lessons from the coronavirus disease 2019 (COVID-19) outbreak in China. FAMA 2020; 323:1239-42.

3. Tran K, Cimon K, Severn M, et al. Aerosol generating procedures and risk of transmission of acute respiratory infections to healthcare workers: a systematic review. PLoS One 2012;7:e35797.

4. Meng L, Qiu H, Wan L, et al. Intubation and ventilation amid the COVID19 outbreak: Wuhan's experience. Anesthesiology 2020;132:1317-32.

5. Sorbello M, El-Boghdadly K, Di Giacinto I, et al.; Società Italiana di Anestesia Analgesia Rianimazione e Terapia Intensiva (SIAARTI) Airway Research Group, and The European Airway Management Society. The Italian coronavirus disease 2019 outbreak: recommendations from clinical practice. Anaesthesia 2020;75:724-32.

6. Matava CT, Yu J, Denning S. Clear plastic drapes may be effective at limiting aerosolization and droplet spray during extubation: implications for COVID-19. Can 7 Anaesth 2020;67:902-4.

7. Canelli R, Connor CW, Gonzalez M, et al. Barrier enclosure during endotracheal intubation [letter]. N Engl 7 Med 2020;382:1957-8.

8. Everington $\mathrm{K}$. Taiwanese doctor invents device to protect US doctors against coronavirus. Taiwan News 2020 Mar. 23. Available: www.taiwannews.com.tw/ en/news/3902435 (accessed 2020 Apr. 8).

9. Lai J. Add a clear plastic drape to PPE - reduce exposure to aerosol and droplets while intubating COVID patients. Twitter 2020 Mar. 16. Available: https:// twitter.com/avecgas/status/1239777525854638080 (accessed 2020 Apr. 9).

10. Kang J, O'Donnell JM, Colaianne B, et al. Use of personal protective equipment among health care personnel: results of clinical observations and simulations. $\mathrm{Am}$ 7 Infect Control 2017;45:17-23.

11. Osei-Bonsu K, Masroor N, Cooper K, et al. Alternative doffing strategies of personal protective equipment to prevent self-contamination in the healthcare setting. Am 7 Infect Control 2019;47:534-9.

12. Tomas ME, Kundrapu S, Thota P. Contamination of health care personnel during removal of personal protective equipment. FAMA Intern Med 2015; 175:1904-10.

13. COVID-19 recommendations during airway manipulation. Toronto: Canadian Anesthesiologists' Society; 2020. Available: www.cas.ca/CASAssets/ Documents/News/Updated-March-25-COVID-19_CAS_Airway_Vsn_4.pdf (accessed 2020 Apr. 6).

14. Cook TM, El-Boghdadly K, McGuire B, et al. Consensus guidelines for managing the airway in patients with COVID-19: guidelines from the Difficult Airway Society, the Association of Anaesthetists the Intensive Care Society, the Faculty of Intensive Care Medicine and the Royal College of Anaesthetists. Anaesthesia 2020;75:785-99.

15. Gardiner C, Veall J, Lockhart S. The use of UV fluorescent powder for COVID®19 airway management simulation training. Anaesthesia 2020; 75:964-5.

16. Birnbach DJ, Rosen LF, Fitzpatrick M, et al. A randomized trial to evaluate a simple strategy to reduce contamination in the operating room. Anesth Analg 2015;120:848-52. 
17. Simpson JP, Wong DN, Verco L, et al. Measurement of airborne particle exposure during simulated tracheal intubation using various proposed aerosol containment devices during the COVID-19 pandemic. Anaesthesia 2020 June 19 [Epub ahead of print]. doi: 10.1111/anae.15188.

18. Begley JL, Lavery KE, Nickson CP, et al. The aerosol box for intubation in coronavirus disease 2019 patients: an in-situ simulation crossover study. Anaesthesia 2020;75:1014-21.

Affiliation: Department of Anesthesiology, Pharmacology and Therapeutics, University of British Columbia, Vancouver General Hospital, Vancouver, BC

Contributors: Peter Rose, John Veall, Navraj Chima, Alana Flexman and Raymond Tang contributed to the conception and design of the study. Peter Rose, John Veall, Elena Vowels, Shruti Chitnis, Alana Flexman and Raymond Tang acquired and interpreted the data. Peter Rose, John Veall and Raymond Tang drafted the manuscript. All authors revised the manuscript for important intellectual content, gave final approval of the version to be published and agreed to be accountable for all aspects of the work.

Data sharing: All data are freely available upon request. Please contact the corresponding author by email for access. As per our institutional policy, data will be kept for 5 years and then destroyed.

Supplemental information: For reviewer comments and the original submission of this manuscript, please see www.cmajopen.ca/content/8/3/ E554/suppl/DC1. 